\title{
Prevalence of pulmonary TB and spoligotype pattern of Mycobacterium tuberculosis among TB suspects in a rural community in Southwest Ethiopia
}

Amare Deribew ${ }^{1,2 *}$, Gemeda Abebe $^{2,3}$, Ludwig Apers $^{4}$, Alemseged Abdissa ${ }^{4}$, Fetene Deribe ${ }^{4}$, Kifle Woldemichael $^{1}$, Chali Jira ${ }^{5}$, Markos Tesfaye ${ }^{6}$, Jafar Shiffa ${ }^{7}$, Abraham Aseffa ${ }^{8}$, Mesele Bezabih $^{3}$, Tadiye Abeje ${ }^{8}$ and Robert Colebunders ${ }^{2,4}$

\begin{abstract}
Background: In Ethiopia where there is no strong surveillance system and state of the art diagnostic facilities are limited, the real burden of tuberculosis (TB) is not well known. We conducted a community based survey to estimate the prevalence of pulmonary TB and spoligotype pattern of the Mycobacterium tuberculosis isolates in Southwest Ethiopia.

Methods: A total of 30040 adults in 10882 households were screened for pulmonary TB in Gilgel Gibe field research centre in Southwest Ethiopia. A total of 482 TB suspects were identified and smear microscopy and culture was done for 428 TB suspects. Counseling and testing for HIV/AIDS was done for all TB suspects. Spoligotyping was done to characterize the Mycobacterium tuberculosis isolates.

Results: Majority of the TB suspects were females (60.7\%) and non-literates (83.6\%). Using smear microscopy, a total of 5 new and 4 old cases of pulmonary TB cases were identified making the prevalence of TB 30 per 100,000. However, using the culture method, we identified 17 new cases with a prevalence of 76.1 per 100,000. There were 4.3 undiagnosed pulmonary TB cases for every TB case who was diagnosed through the passive case detection mechanism in the health facility. Eleven isolates (64.7\%) belonged to the six previously known spoligotypes: T, Haarlem and Central-Asian (CAS). Six new spoligotype patterns of Mycobacterium tuberculosis, not present in the international database (SpolDB4) were identified. None of the rural residents was HIV infected and only 5 (5.5\%) of the urban TB suspects were positive for HIV.

Conclusion: The prevalence of TB in the rural community of Southwest Ethiopia is low. There are large numbers of undiagnosed TB cases in the community. However, the number of sputum smear-positive cases was very low and therefore the risk of transmitting the infection to others may be limited. Active case finding through health extension workers in the community can improve the low case detection rate in Ethiopia. A large scale study on the genotyping of Mycobacterium tuberculosis in Ethiopia is crucial to understand transmission dynamics, identification of drug resistant strains and design preventive strategies.
\end{abstract}

Keywords: Pulmonary TB, Prevalence, Spoligotype, Southwest Ethiopia

\footnotetext{
* Correspondence: amare_deribew@yahoo.com

'Department of Epidemiology, Jimma University, Jimma, Ethiopia

Full list of author information is available at the end of the article
} 


\section{Background}

Tuberculosis (TB) is one of the most challenging communicable diseases for developing countries particularly in Sub-Saharan Africa. A total of 8.8 million incident TB cases have been registered in 96 countries in 2010 [1]. The spread of this disease is fuelled by several factors notably the HIV/AIDS epidemic, low socio-economic status, overcrowding and malnutrition [2-4].

African countries south of the Sahara including Ethiopia are heavily affected by TB. The World Health Organization (WHO) global reports on TB showed that Ethiopia is among the ten top high burden countries in terms of prevalence or incidence cases of TB $[5,6]$.

However, the real burden of TB in Ethiopia is not known due to several reasons. First, there is neither a reliable disease notification system, nor has any regular nation-wide epidemiological survey of TB and HIV been conducted. Second, Ethiopia has poorly developed diagnostic facilities and low health service coverage which might contribute for the very low case detection rate [7]. In the absence of a strong surveillance system, state of the art diagnostic laboratories and high health service coverage, community-based prevalence surveys are good alternatives to estimate the burden of TB and to evaluate the impact of the TB control program. We performed such a survey in a predominantly rural area of Southwest Ethiopia to estimate the prevalence of TB and to characterize the Mycobacterium tuberculosis strains circulating in the community.

\section{Methods}

\section{Study area and study population}

This cross sectional survey was conducted from February to March 2009 in the Gilgel Gibe field research centre, located in Jimma Zone about $260 \mathrm{~km}$ Southwest of Addis Ababa around the reservoir of the Gilgel Gibe hydroelectric dam. The site is bounded by four districts (Woredas): Sekoro, Omo-Nada, Tiro- Afeta and Kersa. In the four Woredas, two urban and eight rural Kebeles (smallest administrative units), were selected as field research center by Jimma University in 2005. They are found within $10 \mathrm{~km}$ of the reservoir of the dam. Since 2005, demographic and AIDS mortality surveillances has been undertaken by Jimma University in the research center. The total population of the field research center is 50156 with the total households of 10,882 . The study population consisted of adults above 14 years of age and TB suspects in the study area.

\section{Sample size calculation and data collection procedures}

The sample size was calculated using the following assumptions: prevalence of TB in rural community of $0.36 \%$ [6], $95 \%$ CI, margin of error of $20 \%$ and non- response rate of $10 \%$. This gave us a total sample of 28917. Since the total adult population in Gilgel Gibe field research centre was 30040 (almost equal to the sample size), all adults $>=15$ years were screened for TB.

A one-page screening questionnaire was prepared in Amharic (local language) to identify all adult TB suspects in the study area. Trained skilled workers who had completed high school education visited each household. If the heads of the households were not available during the visit, the data collectors repeatedly visited the same household 3 times. All adult (age $>=15$ years) with a cough of two weeks or more were considered as TB suspect and given a sputum cup to bring a morning sputum to the nearby school or clinic (appointment centre) the next day. TB suspects were also asked to provide a second "on the spot" sputum sample during their visit to the appointment centre. During their visit, the TB suspects were interviewed about the presence of other symptoms of TB such as fever, their socio-demographic characteristics and perception towards TB using a structured questionnaire. We asked the presence of fever using Affan Oromo (the local language). The study area is malaria endemic and fever is well-known by the local language. Every TB suspect was counseled and asked consent to be tested for HIV. Screening for HIV was done using the KHB test (Shanghai Kehua Bio-Engineering Ltd, Shanghai, China; 2008). A positive sample was retested using the STAT-PAK test (Chembio Diagnostic System Inc, Medford, NY, USA; 2008). Collected sputum samples were transported to Jimma University specialized hospital using a cold box. The same day, sputum smears were examined for the presence of acidfast bacilli (AFB) by an experienced laboratory technician using the standard Ziehl-Neelsen method [8]. Sputum samples were kept at $-20^{\circ} \mathrm{C}$ till transported within a maximum of 5 days to Armauer Hanssen Research Institute (AHRI) in Addis Ababa for culture. At the AHRI, sputum specimens $(2.5-10 \mathrm{ml})$ were processed by the standard N-Acetyl-L-Cysteine-Sodium Hydroxide (NALC-NaOH) method [9] and concentrated at $4000 \times$ $g$ for 15 minutes. The sediment, irrespective of the original sample volume, was reconstituted to $2.5 \mathrm{ml}$ with phosphate buffer $\mathrm{pH} 6.8$, to make the inoculums for the smears and cultures. Two Lowenstein-Jensen slants, one containing $0.75 \%$ glycerol and the other containing $0.6 \%$ pyruvate, were inoculated with the sediment and incubated at $37^{\circ} \mathrm{C}$. Cultures were considered negative when no colonies were seen after 8 weeks incubation period. Isolates were harvested, Deoxyribonucleic Acid (DNA) extracted using a standardized protocol [10] and confirmed as Mycobacterium tuberculosis complex by an inhouse Polymerase Chain Reaction (PCR) [11]. Standard 
spoligotyping method [12] was done generally as described by Kamerbeek and colleagues using a commercially available kit (Isogen Bioscience BV, Maarssen, the Netherlands). The SpolDB4 database [13] and a web-based computer algorithm, Spotclust http://tbinsight.cs.rpi.edu/run_spotclust.html, were used to assign new isolates to families, subfamilies and variants. SpolDB4 assigned names (shared types) were used whenever a spoligo pattern was found in the database. Patterns not found in SpolDB4 were assigned to families and subfamilies by Spotclust. Spoligotypes described only once (non-clustered) in this study and in the SpolDB4 were designated as 'NA' (not assigned). A cluster was defined as two or more isolates from different patients with identical spoligotype patterns.

\section{Quality control measures}

As a quality control measures, we collected adequate recently-discharged mucoid or mucopurulent sputum specimen from TB suspects. The sputum specimens were transported using cold box and immediately examined for the presence of AFB by experienced laboratory technicians based on the standard procedure [8]. All the slides were checked again by the three investigators who are expert in the subject and no discrepancy was observed. Sputum samples for culture were stored at $-20^{\circ} \mathrm{C}$ and then transported in cold boxes within five days to the reference laboratory in AHRI. Culture was done using the Lowenstein-Jensen (LJ) medium based on the standard procedure [11]. For positive samples in the culture, acid fastness was confirmed by Ziehl-Neelsen staining.

\section{Data analysis}

Data were double entered using EpidData software version 3.1(EpidData, Norway, 2006). For analysis, the data was exported to SPSS version 15.0 statistical software (SPSS Inc. Chicago, 2007). Descriptive analysis was done to depict the socio-demographic variables and prevalence of TB. The perception of TB suspects and their health seeking behaviour is published elsewhere [14].

\section{Ethical consideration}

The study protocol was approved by the ethical review committees of Jimma University, Institute of Tropical Medicine (ITM) in Belgium and AHRI. A workshop was held with the local community leaders, Kebele chair persons, and district health office representatives to create awareness about the purpose of the study. Written Consent was obtained from the study participants. New TB cases identified through smear microscopy and culture were immediately referred to the health facility treatment according to the national protocol.

\section{Results}

\section{Characteristics of TB suspects}

A total of 30040 adults in 10882 households were screened for pulmonary TB. Females constituted $50.2 \%$ of the total population. The sex composition of the survey population was similar to the whole population of Gilgel Gibe field research center. From the total adult population in ten Kebeles, 482 TB suspects gave sputum samples, however the samples collected from one Kebele were not used for culture due to delay in transportation and problems with the cold chain. Thus, a total of 428 samples (391 in rural and 91 in urban Kebeles) are presented in this paper. We managed to recruit all the TB suspects and all the TB suspects did also give adequate sputum samples. The mean age of $\mathrm{TB}$ suspects was 41 ( $\mathrm{SD} \pm 16.3$ ); $60.7 \%$ were females; $83.6 \%$ did not had formal education; $71 \%$ were married; $90.4 \%$ were of Oromo ethnicity (Table 1).

The average family size of the TB suspects was 5.5 (SD \pm 2.5$)$. A total of $257(60 \%)$ of the households of TB suspects had no window, 250 (58.4\%) had no separate kitchen and 255 (59.6\%) had two rooms for living. A total of 157 (36.7\%) of the TB suspects were living in close contact with cattle.

More than $70 \%$ of TB suspects had fever in addition to the cough. The mean duration of cough was 13.7 weeks ( $\mathrm{SD} \pm 2.3)$; 154 (36\%) TB suspects had a cough for more than 8 weeks duration.

Table 1 Socio-demographic characteristics of the TB suspects in Gilgel Gibe field research center, Southwest Ethiopia

\begin{tabular}{ll}
\hline Variable & No (\%) \\
\hline Sex & $168(39.3)$ \\
\hline Male & $260(60.7)$ \\
\hline Age, mean(SD) & $41(16.2)$ \\
\hline Marital status & $50(11.7)$ \\
\hline Single & $304(71.0)$ \\
\hline Married & $25(5.8)$ \\
\hline Divorced & $49(11.5)$ \\
\hline Widowed & $387(90.4)$ \\
\hline Religion & $36(8.4)$ \\
\hline Muslim & $5(1.2)$ \\
\hline Orthodox & $70(16.4)$ \\
\hline Protestant & $356(83.6)$ \\
\hline Education
\end{tabular}




\section{Prevalence of TB}

Among the TB suspects, 9 (1.8\%) were found to have TB based on smear microscopy. Five of them were newly identified TB cases; four were already on anti-TB treatment. Thus, the prevalence of TB using smear microscopy was 30 per 100000 populations. A total of 17 new cases of TB were identified by culture of whom 10 (59\%) were females. Including the four TB patients who were on anti-TB treatment and the total population of the nine Kebeles as a denominator $(n=27597)$, the prevalence of TB using the culture method was 76.1 per 100,000 population. The distribution of TB suspects and TB cases detected by culture is depicted in Table 2 . The ratio of passive case detection by smear microscopy in the health facilities $(n=4)$ vs. active case detection by culture $(n=17)$ was $1: 4.3$. This indicated that there were 4.3 undiagnosed pulmonary TB cases for every TB case who was diagnosed through the passive case detection mechanism in the health facility.

\section{Prevalence of HIV among TB suspects}

All the TB suspects were screened for HIV and accepted the test result. All those who were rural residents were negative for HIV. Of the $91 \mathrm{~TB}$ suspects living in a more urban areas, 5(5.5\%) were positive for HIV. Four of them were located in Deneba where there were many immigrants working at the hydroelectric power plant.

\section{Spoligotyping}

A total of 14 spoligotypes were identified among the 17 Mycobacterium tuberculosis isolates. Six isolates (35.3\%) had new and unique spoligotypes while 11 isolates (64.7\%) belonged to the six previously known spoligotypes: T, Haarlem and Central-Asian. The Six isolates were grouped into three clusters ( 2 isolates per cluster), while the remaining 11 strains did not cluster. Of the eleven strains that did not cluster, six were new, hence represented the true orphans in the study sample. The remaining five of the unclustered isolates included strains with label T3_ETH, H4, T1 and H3-T3. One isolate lacked hybridization to spacer 33-36 and 40, which is characteristic of strains previously classified as Mycobacterium africanum genotype [15,16] (Figure 1).

\section{Discussion}

Our study determined the prevalence of pulmonary TB in a predominantly rural community of Southwest Ethiopia. The prevalence of TB using smear microscopy was lower than the prevalence reported in Addis Ababa (189 per 100,000) [17], South Ethiopia (78 per 100,000) [18] and North Ethiopia (80 per 100,000) [19]. The low prevalence of HIV infection and the absence of overcrowding in this predominantly rural community could explain the low prevalence of pulmonary TB. Overcrowding and the HIV epidemic are indeed the driving forces for the spread of TB [20,21]. Using the culture method, we identified 4.3 undetected infectious TB cases for every TB patient diagnosed through the passive case detection mechanism of the health facilities. These large number of undiagnosed TB cases can transmit the disease to others particularly family members. Similar to other studies $[8,22,23]$, we identified more undiagnosed female than male patients. Lack of empowerment to seek early health care and low knowledge of women about the cause of TB [14] could be the reasons for this high number of undiagnosed female TB patients in the community.

The spoligotyping revealed that the majority (29.4\%) of the strains in our samples were of the $\mathrm{T}$ family, a finding which is in agreement with those of earlier studies from other parts of Ethiopia [24]. The T3-ETH was reported previously to be frequent in Ethiopia as MDR strains [25]. This clone which was characterized by low-banding IS6110-RFLP was identified in 1995 and represented $36.2 \%$ of isolates [25]. It has been described in the international database as a spoligotype

Table 2 Distribution of TB suspects in the study Kebeles of Gilgel Gibe Field Research Center, Southwest Ethiopia

\begin{tabular}{llllllll}
\hline Kebele & \multicolumn{2}{l}{ Total population surveyed } & \multicolumn{2}{l}{ TB suspects } & Number TB Cases identified by culture \\
\cline { 2 - 7 } & Male & Female & Total & Male & Female & Total & \\
\hline Siba & 1484 & 1409 & 2893 & 12 & 25 & 37 & $4(10.8 \%)$ \\
\hline Asendabo & 2226 & 2267 & 4493 & 18 & 23 & 41 & 0 \\
\hline Burka & 1806 & 1733 & 3539 & 32 & 32 & 64 & $2(3.1 \%)$ \\
\hline Kejello & 1662 & 1747 & 3409 & 16 & 31 & 47 & 0 \\
\hline Koticha & 1150 & 1192 & 2342 & 19 & 24 & 43 & 0 \\
\hline Ayno & 1704 & 1678 & 3382 & 17 & 47 & 64 & $6(9.4 \%)$ \\
\hline Enkure & 1015 & 1033 & 2048 & 16 & 31 & 47 & $2(4.3 \%)$ \\
\hline Bore & 1051 & 1014 & 2065 & 21 & 14 & 35 & $1(2.9 \%)$ \\
\hline Deneba & 1622 & 1804 & 3426 & 17 & 33 & 50 & $2(4.0 \%)$ \\
\hline Total & $\mathbf{1 3 7 2 0}$ & $\mathbf{1 3 8 7 7}$ & $\mathbf{2 7 5 9 7}$ & $\mathbf{1 9 3}$ & $\mathbf{2 6 0}$ & $\mathbf{4 2 8}$ & $\mathbf{1 7 ( 3 . 9 \% )}$ \\
\hline
\end{tabular}




\begin{tabular}{|c|c|c|c|}
\hline SIT No Binary format & & SpolDB4 & $\overline{\text { Cluster }}$ \\
\hline 777 & & $\mathrm{H} 4$ & I \\
\hline 25 & & CAS1_Delhi & II \\
\hline 37 & & $\mathrm{~T} 3$ & III \\
\hline NA & & NA & \\
\hline NA & & NA & \\
\hline NA & & NA & \\
\hline NA & & NA & \\
\hline NA & & NA & \\
\hline NA & & NA & \\
\hline 149 & & T3_ETH & \\
\hline 817 & & $\mathrm{H} 4$ & \\
\hline 1877 & & $\mathrm{~T} 1$ & \\
\hline 53 & & $\mathrm{~T} 1$ & \\
\hline 36 & & H3_T3 & \\
\hline
\end{tabular}

Figure 1 Spoligotype pattern of $\boldsymbol{M}$. tuberculosis. There were 11 isolates with distinct spoligoype pattern. NA indicates strains not found in SpolDb4 data base; Cluster represents group of two or more patient isolates that shared the same spoligoype pattern; cluster I, II and III each represent 2 isolates. Other spoligotype patterns represent single patient isolate. SIT = Spoligo-international type, CAS = Central-Asian.

that is also common in Ethiopia, Kenya and Libya [13]. The second most prevalent lineage was Harlem $(\mathrm{H})$ (23.5\%) which is commonly found in Poland, Saudi Arabia, Tunisia, Vietnam and Venezuela [13]. Two strains in the study sample were of the Central-Asian (CAS) family which has been described as essentially localized in the Middle East and Central Asia and preferentially in India [26].

Our study identified six new spoligotype patterns of Mycobacterium tuberculosis which were not present in the international database. This indicates that there is little information about $M$. tuberculosis strains circulating in the area. Since only a small number of isolates were characterized in our study, it is not possible to appreciate the diversity of Mycobacterium tuberculosis circulating in the area. More isolates and a wider geographical coverage is required to have a detail insight about the Mycobacterium tuberculosis strains circulating in Southwest Ethiopia.

Our study is the first of its kind in Southwest Ethiopia to assess the prevalence of TB and HIV at community level. The study tried to identify all TB suspects using repeated house to house visits. However, the study has its limitations. Fifty-four sputum samples were not examined due to an unreliable cold chain which could affect the correct estimation of the prevalence of pulmonary TB. Moreover, storage of the sputum specimens at $-20^{\circ} \mathrm{C}$ might have an effect on the viability of $\mathrm{Myco}$ bacterium tuberculosis. This could under-estimate the culture positivity rate in our study. As a result of the small number of the isolates, a full spectrum of the Mycobacterium tuberculosis strains could not be documented.

In conclusion, the prevalence of $\mathrm{TB}$ in the rural community of Southwest Ethiopia is low. There are large numbers of undiagnosed TB cases in the community. However, the number of sputum smear-positive cases was very low and therefore the risk of transmitting the infection to others may be limited. The Ethiopian TB control program should design a strategy to improve case detection rates through the provision of tailored health education messages and involvement of lower health cadres at the grassroots level. A community-based randomized trial in Ethiopia showed that involvement of lower health cadres and health education improved the case detection rate of TB significantly (122.2\% in the intervention villages vs. $69.4 \%$ in the control villages) [27]. Additional training for these lower health cadres about case detection and referral of TB cases could help the Ethiopian government to improve the case detection rate and achieve the TB related millennium development goals. We recommend researchers to conduct a large scale community-based study on the molecular epidemiology of $\mathrm{TB}$ to determine the strain lineage and drug sensitivity pattern in Ethiopia. A large scale study on the genotyping of Mycobacterium tuberculosis in Ethiopia is crucial to understand transmission dynamics, 
identification of drug resistant strains and design preventive strategies.

\section{Acknowledgements}

We acknowledge the study participants and the community for the information provided. The study was funded by Directorate General for Development Cooperation (DGDC) through the Flemish Interuniversity council (VLIR-UOS).

\section{Author details \\ 'Department of Epidemiology, Jimma University, Jimma, Ethiopia. ${ }^{2}$ University of Antwerp, Antwerp, Belgium. ${ }^{3}$ Department of Laboratory Sciences and pathology, Jimma University, Jimma, Ethiopia. ${ }^{4}$ Institute of Tropical Medicine, Antwerp, Belgium. ${ }^{5}$ Department of Health service management and Planning, Jimma University, Jimma, Ethiopia. ${ }^{6}$ Department of psychiatry, Jimma University, Jimma, Ethiopia. ${ }^{7}$ Department of Internal Medicine, Jimma University, Jimma, Ethiopia. ${ }^{8}$ Armour Hansen Research Institute, Addis Ababa, Ethiopia.}

\section{Authors' contributions}

$\mathrm{AD}$ was involved in the conception and design of the study, coordinated the field work, analyzed the data and drafted the manuscript. GA was involved in the conception, design of the study, field work and review of the article. LA was involved in the design and reviewed the article. AA, FD, $\mathrm{KW}, \mathrm{CJ}, \mathrm{MT}, J \mathrm{~S}, \mathrm{AA}$ and $\mathrm{MB}$ participated in the design, field work and reviewed the article. TA did the laboratory work and reviewed the article. $\mathrm{RC}$ participated in the design, critically reviewed and approved the article. All authors read and approved the final manuscript.

\section{Competing interests}

The authors declare that they have no competing interests.

Received: 30 July 2011 Accepted: 13 March 2012

Published: 13 March 2012

\section{References}

1. WHO: Global Tuberculosis Report. WHO Report 2011.[http://www.who.int/ tb/publications/global_report/en/].

2. Harries AD, Dye C: Tuberculosis. Annals of Tropical Medicine and Parasitology 2006, 5(6):415-431.

3. Spence DP, Hotchkiss J, Williams CS, et al: Tuberculosis and poverty. BMJ 1993, 307(6907):759-761.

4. Bartlett JG: Tuberculosis and HIV infection: partners in human tragedy. Infect Dis 2007, 196(Suppl 1):S124-S125.

5. WHO: Global tuberculosis control: epidemiology, strategy, financing: WHO report 2009. WHO, Geneva; WHO/HTM/TB/2009.411.

6. WHO: Global tuberculosis control: epidemiology, strategy, financing: WHO report 2010. WHO, Geneva; WHO/HTM/TB/2009.411.

7. Ministry of Health, Ethiopia: TB, leprosy and TB/HIV prevention and control program manual, fourth edition. Addis Ababa, Ethiopia; 2007 [http://www.moh.gov.et]

8. International Union Against Tuberculosis and Lung Disease: Sputum examination for tuberculosis by direct microscopy in low income countries. 5 edition. Paris, France: IUATLD; 2000.

9. Cruciani M, Scarparo C, Malena M, Bosco O, Serpelloni G, Mengoli C: Metaanalysis of BACTEC MGIT 960 and BACTEC 460, with or without solid media, for detection of mycobacteria. J Clin Microbiol 2004, 42:2321-2325.

10. Van Embden JD, Cave MD, Crawford JT, Dale JW, Eisenach KD, et al: Strain identification of Mycobacterium tuberculosis by DNA fingerprinting: recommendations for a standardized methodology. J Clin Microbiol 1993, 31:406-409.

11. Muhumuza J, Asiimwe BB, Kayes S, Mugyenyi R, Whalen $C$, et al: Introduction of an in-house PCR for routine identification of M. tuberculosis in a low-income country. Int J Tuberc Lung Dis 2006, 10:1262-1267.
12. Kamerbeek J, Schouls L, Kolk A, van Agterveld M, van Soolingen D, et al Simultaneous detection and strain differentiation of Mycobacterium tuberculosis for diagnosis and epidemiology. J Clin Microbiol 1997, 35:907-914.

13. Brudey K, Driscoll JR, Rigouts $L$, Prodinger WM, Gori A, et al: Mycobacterium tuberculosis complex genetic diversity: mining the fourth international spoligotyping database (SpolDB4) for classification, population genetics and epidemiology. BMC Microbiol 2006, 6:23.

14. Abebe G, Deribew A, Apers L, Woldemichael K, Shiffa J, et al: Knowledge, Health Seeking Behavior and Perceived Stigma towards Tuberculosis among Tuberculosis Suspects in a Rural Community in Southwest Ethiopia. PLoS One 2010, 5(10):e13339..

15. Niemann S, Kubica T, Bange FC, Adjei O, Browne EN, et al: The species Mycobacterium africanum in the light of new molecular markers. J Clin Microbiol 2004, 42:3958-3962.

16. Niemann S, Rusch-Gerdes S, Joloba ML, Whalen CC, Guwatudde D, et al Mycobacterium africanum subtype II is associated with two distinct genotypes and is a major cause of human tuberculosis in Kampala, Uganda. J Clin Microbiol 2002, 40:3398-3405.

17. Demissie $M$, Zenebere $B$, Berhane $Y$, Lindtjorn $B$ : A rapid survey to determine the prevalence of smear-positive tuberculosis in Addis Ababa. Int J Tuberc Lung Dis 2002, 6(7):580-584.

18. Shargie B, Yassin M, Lindtjorn B: Prevalence of smear-positve pulmonary tuberculosis in a rural district of Ethiopia. Int J Tuberc Lung Dis 2006, 10(1):87-92

19. Yimer S, Holm-Hansen C, Yimaldu T, Bjune G: Evaluating an active case finding strategy to identify smear-positive tuberculosis in rural Ethiopia. Int J Tuberc Lung Dis 2010, 13(11):1399-1404.

20. Lonnroth K, Jaramillo E, Williams BG, Dye C, Raviglione M: Drivers of tuberculosis epidemics: the role of risk factors and social determinants. Soc Sci Med 2009, 68(12):2240-2246.

21. Dye C, Maher D, Weil D, Espinal M, Raviglione M: Targets for global tuberculosis control. Int J Tuberc Lung Dis 2006, 10(4):460-462.

22. Subramani R, Radhakrishna S, Frieden $T R$, et al: Rapid decline in prevalence of pulmonary tuberculosis after DOTS implementation in a rural area of South India. Int J Tuberc Lung Dis 2008, 12:916-920.

23. Hamid Salim MA, Declercq E, Van Deun A, Saki KA: Gender difference in tuberculosis: a prevalence survey done in Bangladesh. Int J Tuberc Lung Dis 2004, 8:952-957.

24. Agonafir M, Lemma E, Wolde-Meskel D, Goshu S, Santhanam A, et al: Phenotypic and genotypic analysis of multidrug-resistant tuberculosis in Ethiopia. Int J Tuberc Lung Dis 2010, 14:1259-1265.

25. Hermans PW, Messadi F, Guebrexabher H, van Soolingen D, de Haas PE, et al: Analysis of the population structure of Mycobacterium tuberculosis in Ethiopia, Tunisia, and The Netherlands: usefulness of DNA typing for global tuberculosis epidemiology. J Infect Dis 1995, 171:1504-1513.

26. Bhanu NV, van Soolingen D, van Embden JD, Dar L, Pandey RM, et al: Predominace of a novel Mycobacterium tuberculosis genotype in the Delhi region of India. Tuberculosis (Edinb) 2002, 82:105-112.

27. Datiko DG, Lindtjørn B: Health Extension Workers Improve Tuberculosis Case Detection and Treatment Success in Southern Ethiopia: A community Randomized Trial. PLoS One 2009, 4(5):e5443.

\section{Pre-publication history}

The pre-publication history for this paper can be accessed here: http://www.biomedcentral.com/1471-2334/12/54/prepub

doi:10.1186/1471-2334-12-54

Cite this article as: Deribew et al:: Prevalence of pulmonary TB and spoligotype pattern of Mycobacterium tuberculosis among TB suspects in a rural community in Southwest Ethiopia. BMC Infectious Diseases 2012 12:54. 